\title{
Systematic mapping review of the factors influencing physical activity and sedentary behaviour in ethnic minority groups in Europe: a DEDIPAC study
}

Lars Jørun Langøien ${ }^{1}$, Laura Terragni ${ }^{2}$, Gro Rugseth', Mary Nicolaou ${ }^{3}$, Michelle Holdsworth ${ }^{4}$, Karien Stronks ${ }^{3}$, Nanna Lien ${ }^{5}$, Gun Roos ${ }^{6^{*}}$ (i) and on behalf of the DEDIPAC consortium

\begin{abstract}
Background: Physical activity and sedentary behaviour are associated with health and wellbeing. Studies indicate that ethnic minority groups are both less active and more sedentary than the majority population and that factors influencing these behaviours may differ. Mapping the factors influencing physical activity and sedentary behaviour among ethnic minority groups living in Europe can help to identify determinants of physical activity and sedentary behaviour, research gaps and guide future research.

Methods: A systematic mapping review was conducted to map the factors associated with physical activity and sedentary behaviour among ethnic minority groups living in Europe (protocol PROSPERO ID = CRD42014014575). Six databases were searched for quantitative and qualitative research published between 1999 and 2014. In synthesizing the findings, all factors were sorted and structured into clusters following a data driven approach and concept mapping.

Results: Sixty-three articles were identified out of 7794 returned by the systematic search. These included 41 quantitative and 22 qualitative studies. Of these 58 focused on physical activity, 5 on both physical activity and sedentary behaviour and none focused on sedentary behaviour. The factors associated with physical activity and sedentary behaviour were grouped into eight clusters. Social \& cultural environment $(n=55)$ and Psychosocial (39) were the clusters containing most factors, followed by Physical environment \& accessibility (33), Migration context (15), Institutional environment (14), Social \& material resources (12), Health and health communication (12), Political environment (3). An important finding was that cultural and religious issues, in particular those related to gender issues, were recurring factors across the clusters.
\end{abstract}

Conclusion: Physical activity and sedentary behaviour among ethnic minority groups living in Europe are influenced by a wide variety of factors, especially informed by qualitative studies. More comparative studies are needed as well as inclusion of a wider spectrum of the diverse ethnic minority groups resettled in different European countries. Few studies have investigated factors influencing sedentary behaviour. It is important in the future to address specific factors influencing physical activity and sedentary behaviour among different ethnic minority groups in order to plan and implement effective interventions.

Keywords: Physical activity, Sedentary behaviour, Factors, Ethnic minority groups, Europe, Migrants, Immigrants

\footnotetext{
* Correspondence: Gun.Roos@sifo.hioa.no

${ }^{6}$ Consumption Research Norway - SIFO, Oslo and Akershus University

College of Applied Sciences, Oslo, Norway

Full list of author information is available at the end of the article
} 


\section{Background}

Low levels of physical activity (PA) and high levels of sedentary behaviour (SB) are associated with obesity and non-communicable diseases (NCDs), including type 2 diabetes and cardiovascular diseases (WHO, 2014). Enhancing the levels of $\mathrm{PA}$ and reducing the levels of $\mathrm{SB}$ can prevent and to some extent treat NCDs. Studies indicate that some ethnic minority groups are less active and more sedentary than the majority population and that factors influencing these behaviours may differ (e.g. [1-3]). In Europe, reviews have been undertaken on obesity and PA among a limited number of ethnic minority or migrant groups. These include reviews of PA in North African migrants [4] and South Asian women in different Western countries [5]. These reviews mainly focused on levels of PA, and indicated that information on barriers and facilitators is limited. However, to develop effective interventions that also reach ethnic minority groups information about factors influencing PA and SB is necessary. To our knowledge there are no systematic reviews on factors influencing PA and SB in ethnic minority groups across Europe. The ethnic minority groups in Europe are quite diverse in terms of size, country of origin and migration patterns, and there are substantial variations between countries [6]. In recent years the number of asylum seekers have increased and the three largest groups have been from Syria, Afghanistan and Iraq [7]. Refugees, asylum seekers and migrants seem to be at risk for worse health outcomes including NCDs [8]. As the migrant composition in Europe is changing and growing, gathering knowledge on factors influencing PA and SB of migrants and identifying gaps in the literature is crucial for assessing the needs of these populations and planning interventions.

The aim of this study was to systematically review the literature that has identified factors influencing PA and $\mathrm{SB}$ across the life-course among ethnic minority groups living in Europe, uncover gaps in the literature and suggest priorities for future research. This review is part of the work performed on ethnic minority groups in the DEDIPAC (Determinants of Diet and Physical Activity) Knowledge Hub [9]. DEDIPAC $\mathrm{KH}$ is a European research network aiming at understanding the 'causes of the causes' of diet, physical activity and sedentary behaviour. The evidence from this review also feeds into a DEDIPAC study of factors influencing diet and PA/SB behaviours in ethnic minority groups in Europe [10].

\section{Methods}

We conducted a systematic mapping review to find both quantitative and qualitative published literature. According to Grant and Booth [11] mapping reviews are suited to map out the existing literature to identify tendencies and gaps in the research literature to commission future research. The review protocol was registered with PROSPERO (ID = CRD42014014575).

The stages of the data selection process are presented in Fig. 1. The search was conducted in the following electronic databases: MEDLINE, EMBASE (Ovid), Web of Science, Cochrane Library, CINAHL, Psychinfo (Ovid). The databases were searched from 1999 to 2014. This time period was chosen because we anticipated that factors identified before 1999 would be referred to in more recent literature. The citation follow-up technique was used to identify studies that had not been identified through the systematic search of the databases. Additionally, experts in the subject area, researchers working in the fields of physical activity and sedentary behaviour, ethnicity and health were contacted to identify relevant studies that might have been missed in the search of electronic databases.

Ethnic minority group is a concept used for very heterogeneous groups that may share minority status in their country of residence due to ethnicity, place of birth, language, religion, citizenship as well as other cultural differences. This definition may include groups from newly arrived immigrants to (minority) groups that have been part of a country's history, for instance the Roma and Sami people. Within the DEDIPAC consortium we reached an agreement on what we meant by 'Ethnic minority groups', who were defined as immigrants/populations of immigrant background (not differentiating based on their migration status) from low and middle income countries, population groups from the former Eastern Bloc countries who migrate to other parts of Europe and minority indigenous populations in Europe [9]. We decided to focus on these ethnic minority groups because they are more likely to be of lower socioeconomic status.

\section{Inclusion and exclusion criteria}

Observational and intervention studies using quantitative and/or qualitative methods examining physical activity and sedentary behaviour among ethnic minority groups in Europe were included. In addition, studies that identified an association between a factor (including correlates, predictors, moderators, determinants and mediators) and physical activity or sedentary behaviour of ethnic minority groups living in Europe were included. All age groups were included.

Studies that analysed physical activity or sedentary behaviour as a confounder in a relationship between ethnicity and disease, including studies that explored whether ethnicity is a factor influencing physical activity or sedentary behaviour without explanation were excluded because we were interested in studies that contained relevant information on factors. Studies only presenting descriptive information about physical activity or sedentary behaviour, or just describing levels of activity according to 


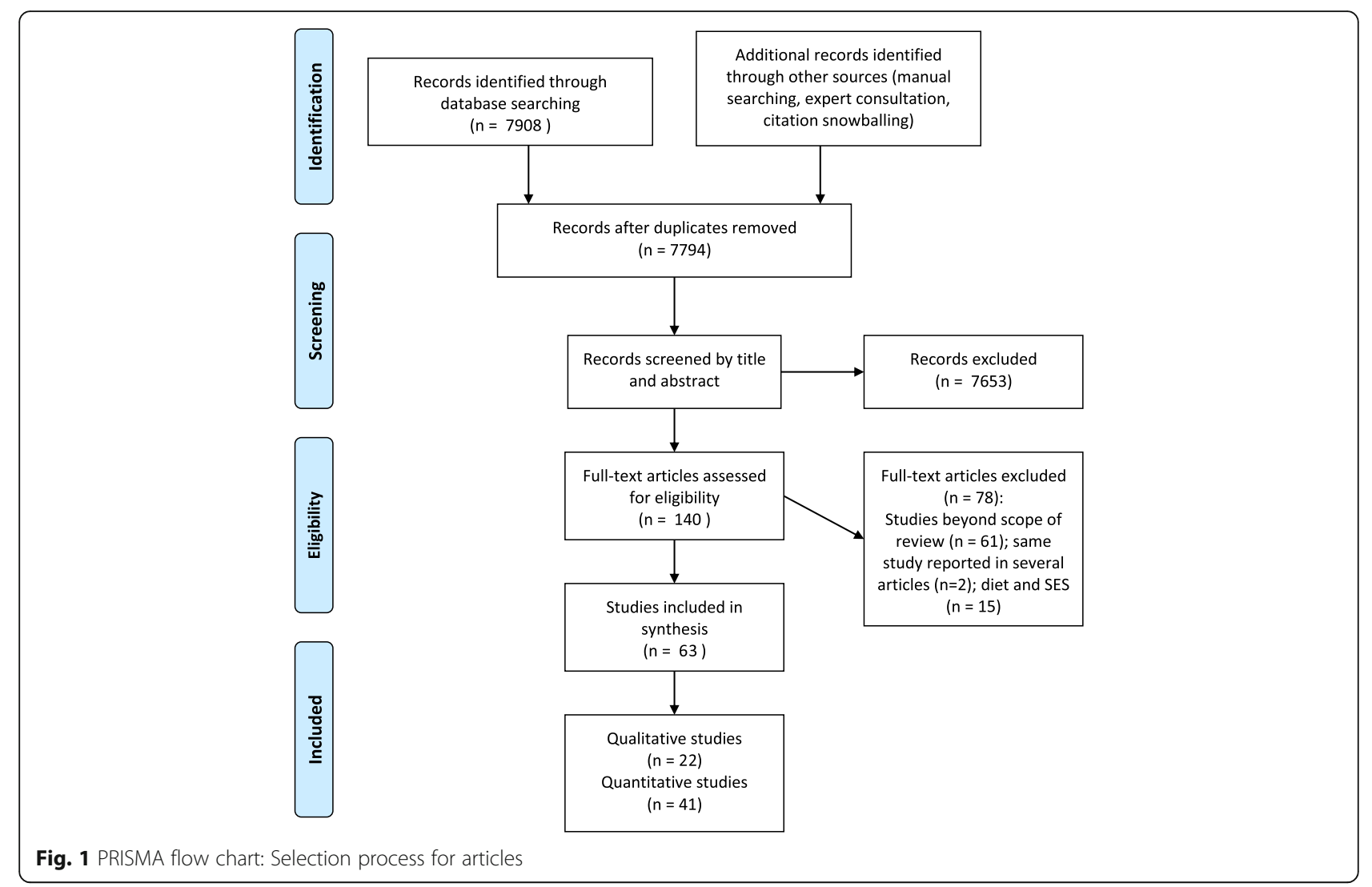

belonging to an ethnic minority group without providing any explanation were also excluded. As were non-human studies and laboratory based studies.

\section{Search strategy and study selection}

A search strategy was constructed to identify studies with the use of free text word and MESH terms, which was modified for each database. The search strategy was guided by search terms within three concepts: physical activity and/or sedentary behaviour; ethnic minority group; and Europe (as the setting). The complete strategy is shown in Additional file 1. Retrieved literature was stored in an Endnote database and duplicate entries were deleted.

To ensure accuracy and consistency of data extraction two reviewers (LJL and LT) independently screened titles and abstracts of identified studies for relevance, according to the review inclusion criteria. Spot checks were conducted on a sample of screened sources to assess the extent of agreement between reviewers. All retrieved full texts papers were reviewed by two of the team of four independent researchers (GrR and LJL or LT and GR).

\section{Data extraction and synthesis}

Data from papers included in the full text review phase were extracted by four independent researchers (LJL, LT,
GrR and GR). An extraction tool was developed to collate data which was used to identify general information and study characteristics (author, year of publication, country, and study design, sample size), population characteristics (gender, age, country of origin, years since migration, acculturation, education, migration history, and other relevant demographics).

Quality assessment of quantitative and qualitative studies was undertaken using established quality assessment criteria for evaluating primary research papers [12]. The quality of articles included after the full text review were assessed by two of the team of four independent researchers involved in data extraction. The quality assessment scores given to the included studies are included in Tables 1 and 2 .

Common methodologies for identifying and grouping factors were used as for a similar review on dietary determinants of ethnic minority groups [13]. First, all factors from the included studies were identified. Then these factors were sorted into clusters according to how they were seen to relate to each other, following a data driven approach [14]. The clustering was part of a larger concept mapping process with the aim of developing a systembased framework of factors influencing dietary behaviour and physical activity/sedentary behaviour in the general European population [15-17] and in ethnic minority 


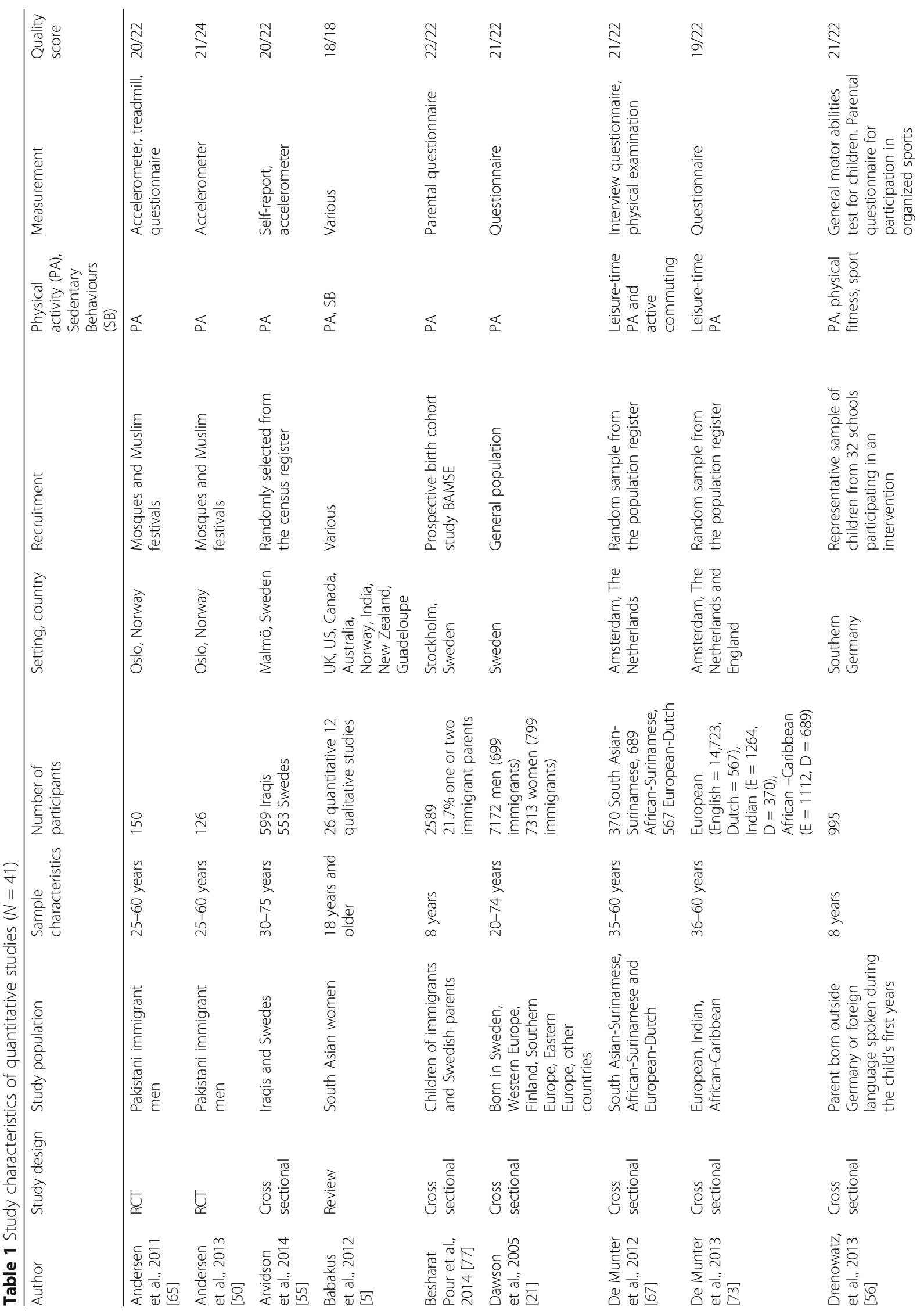




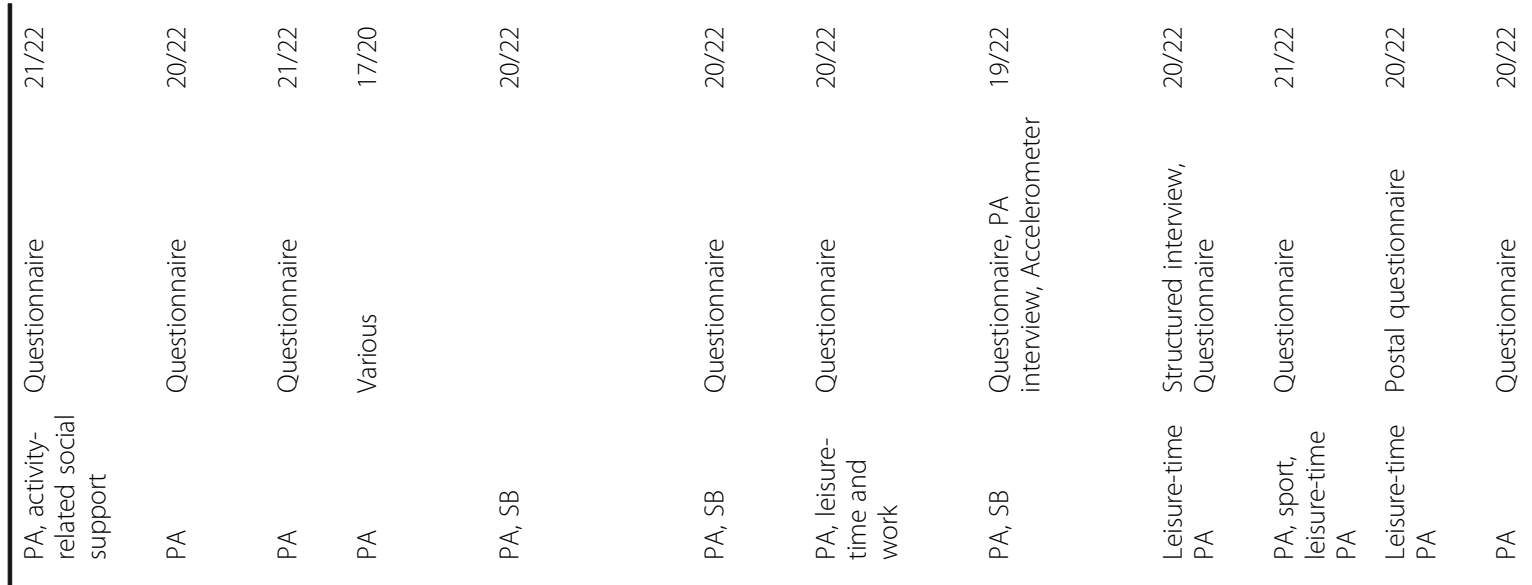

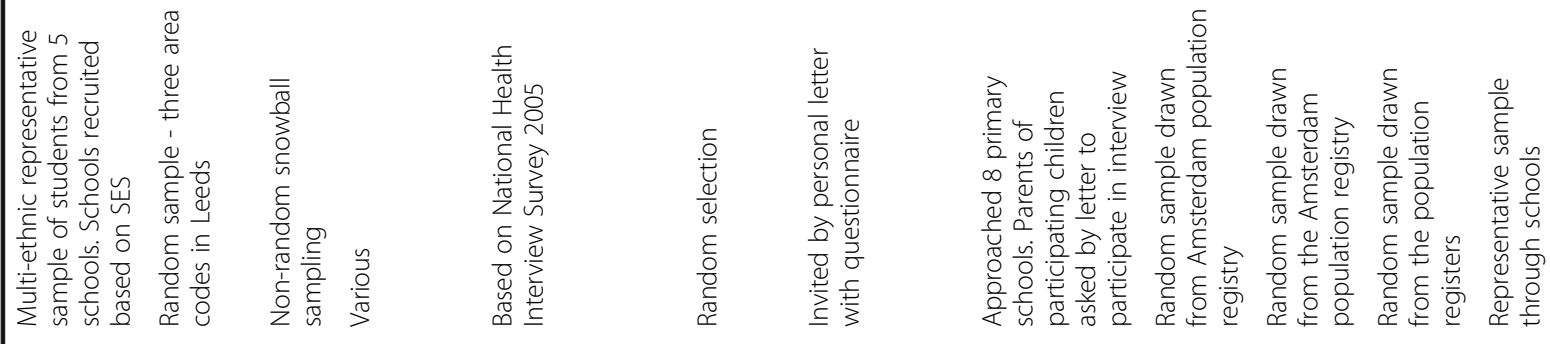

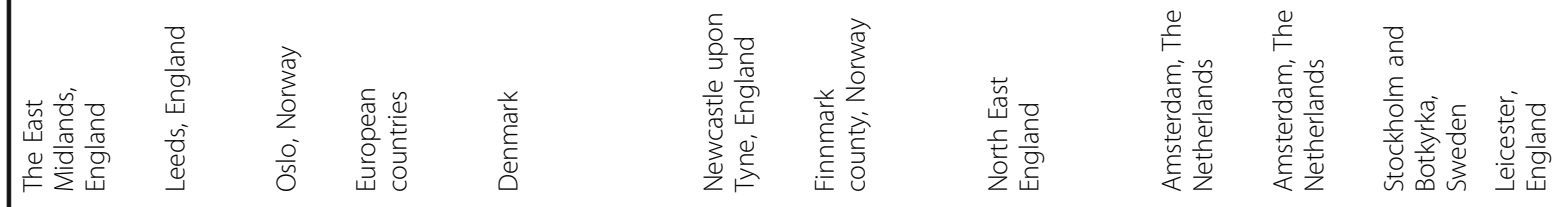

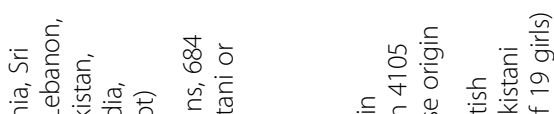

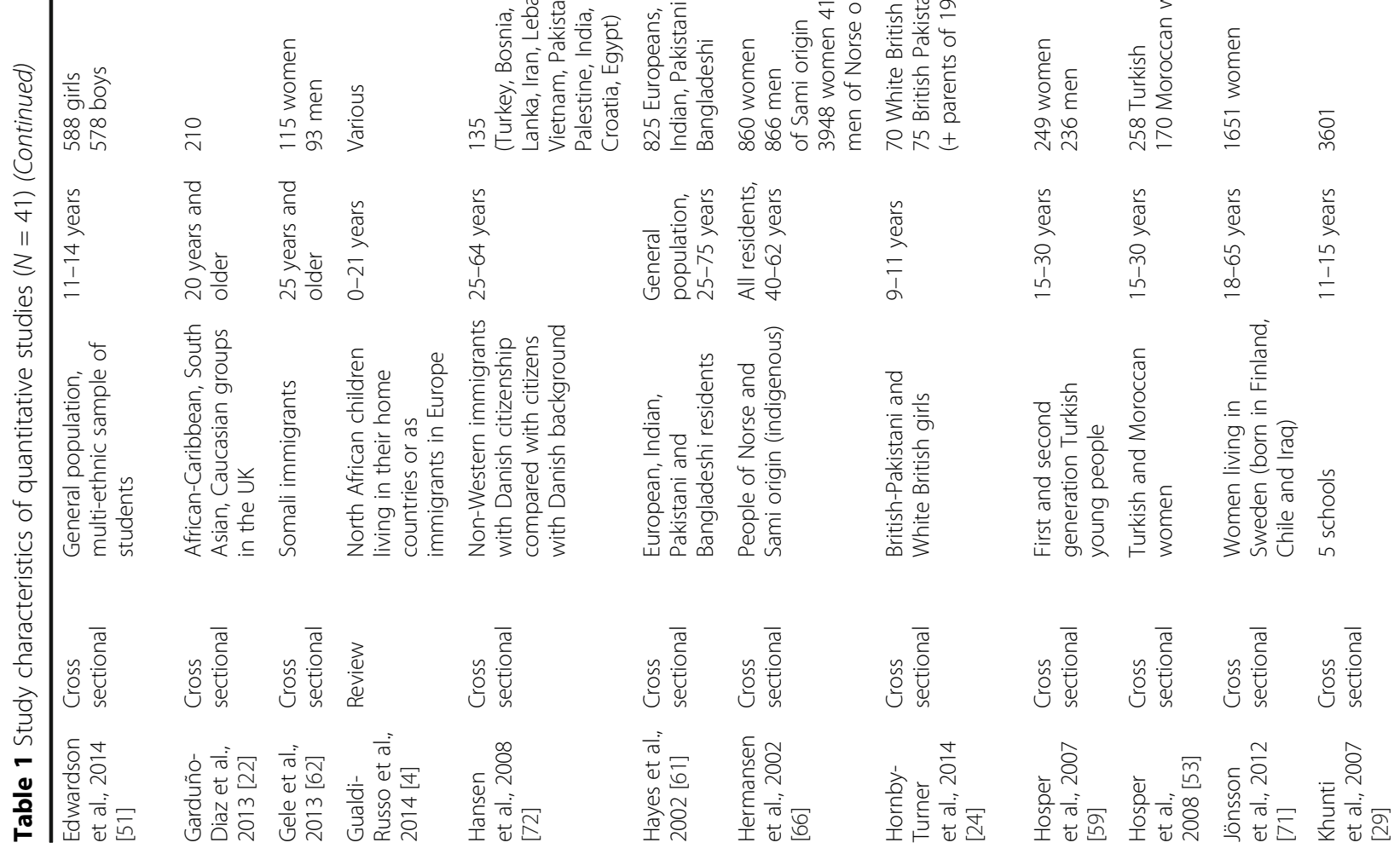




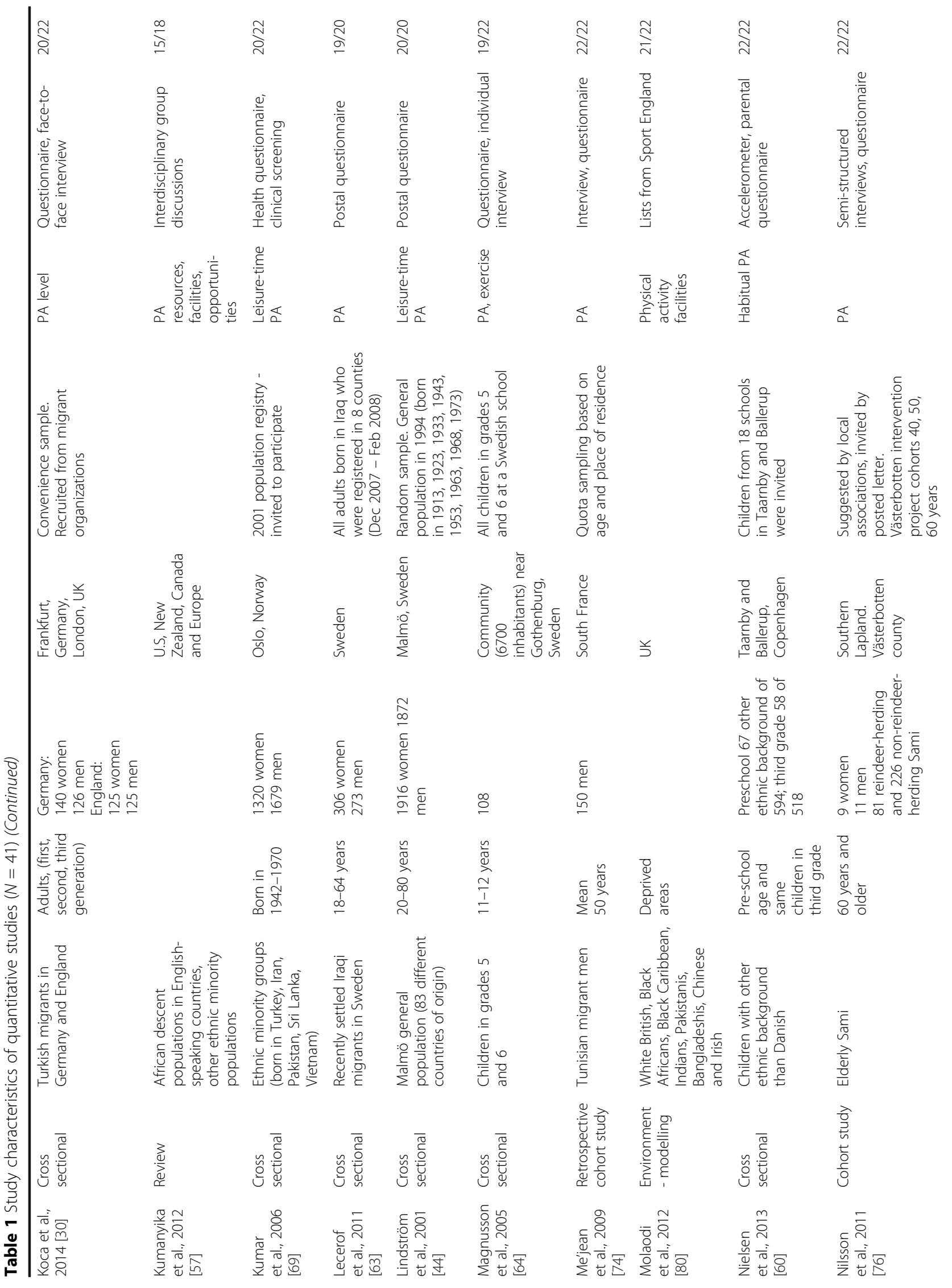




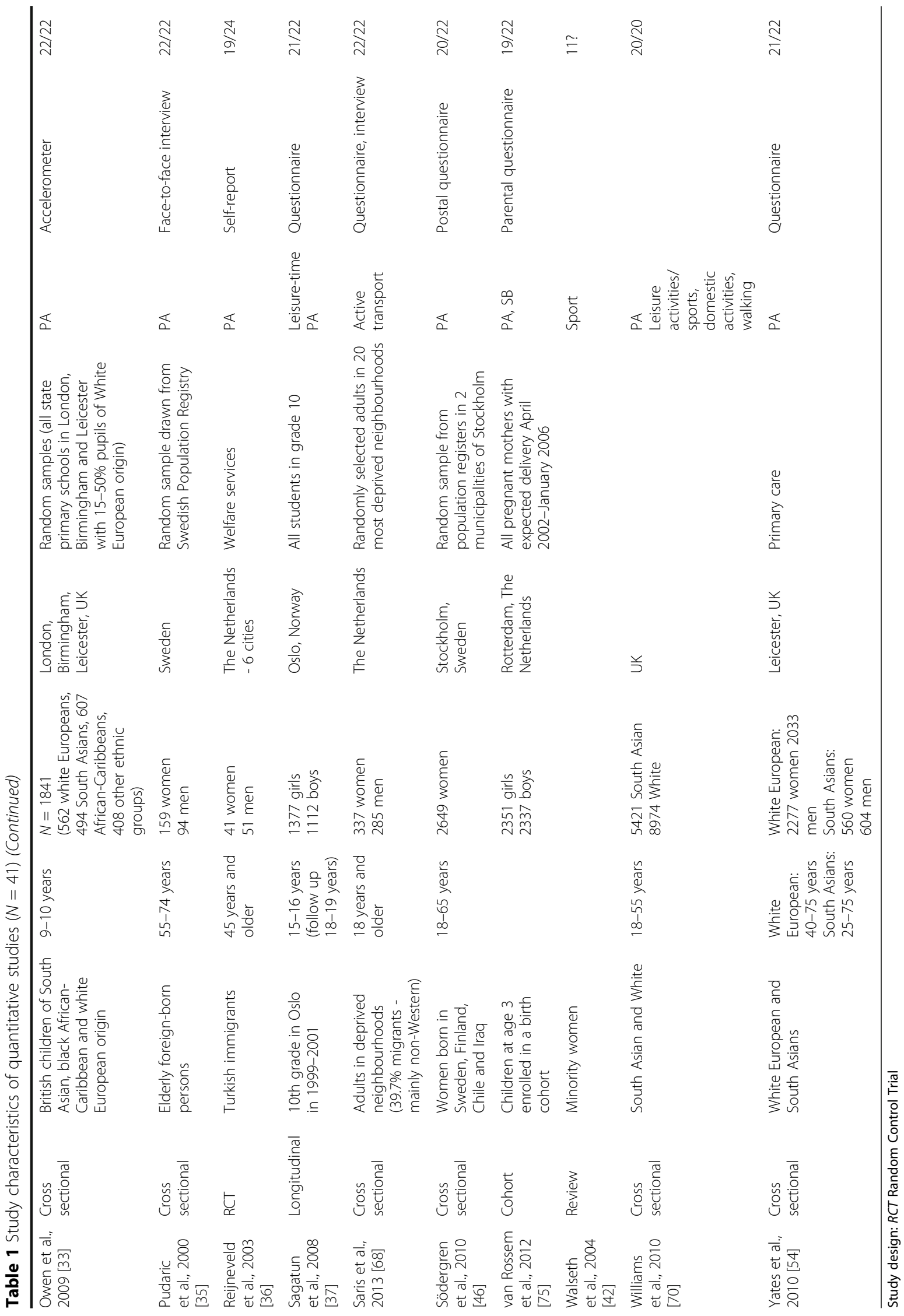




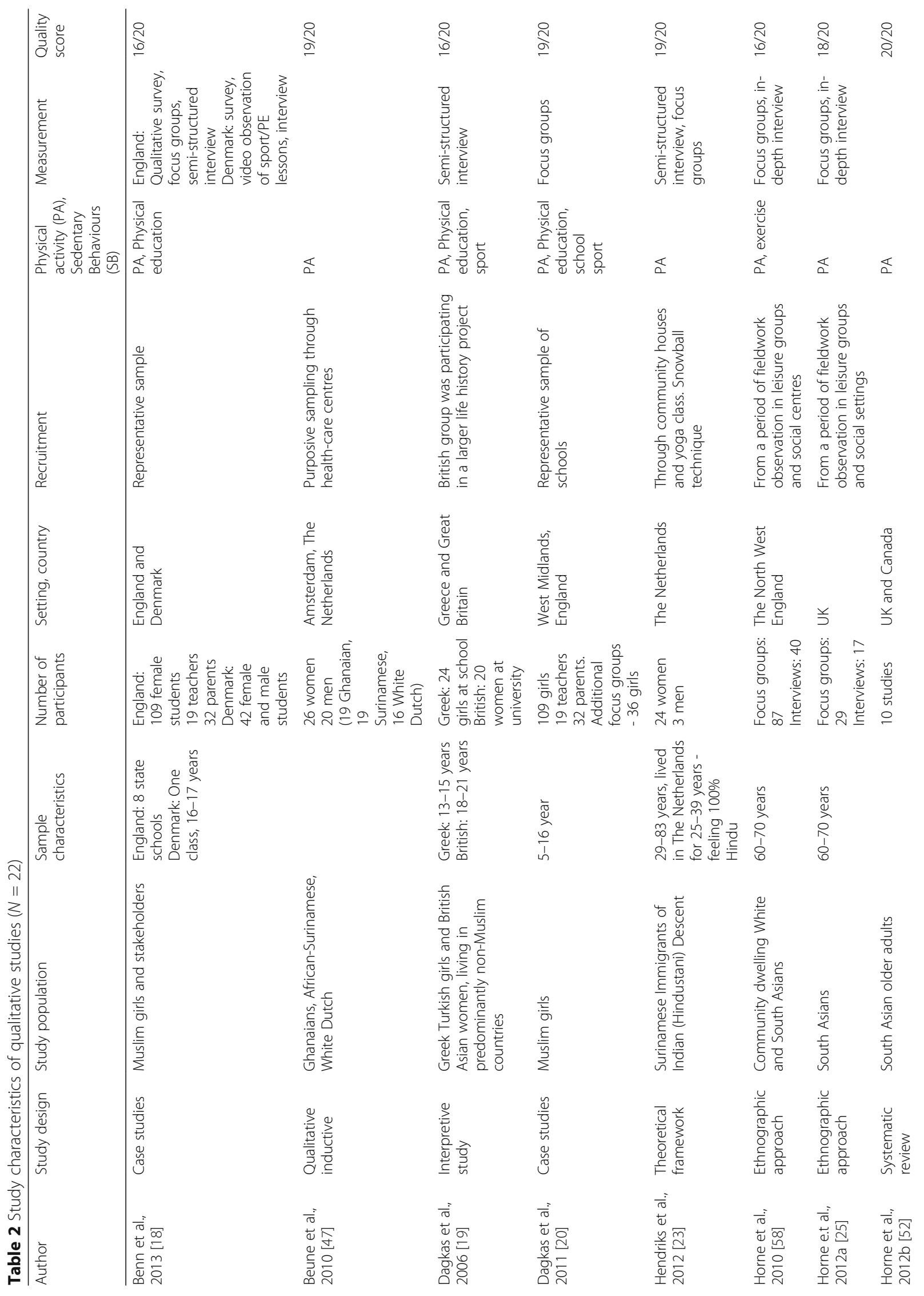




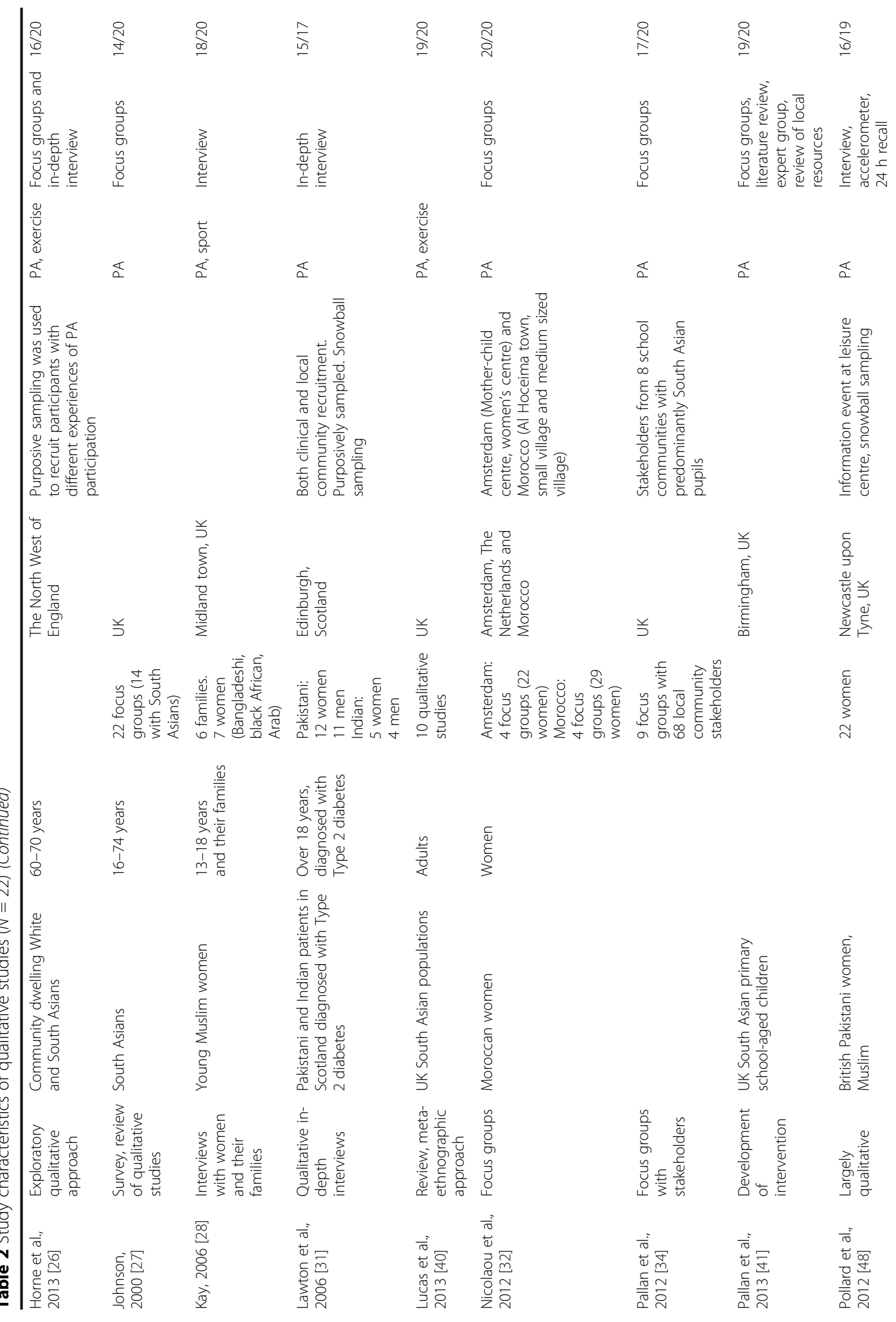




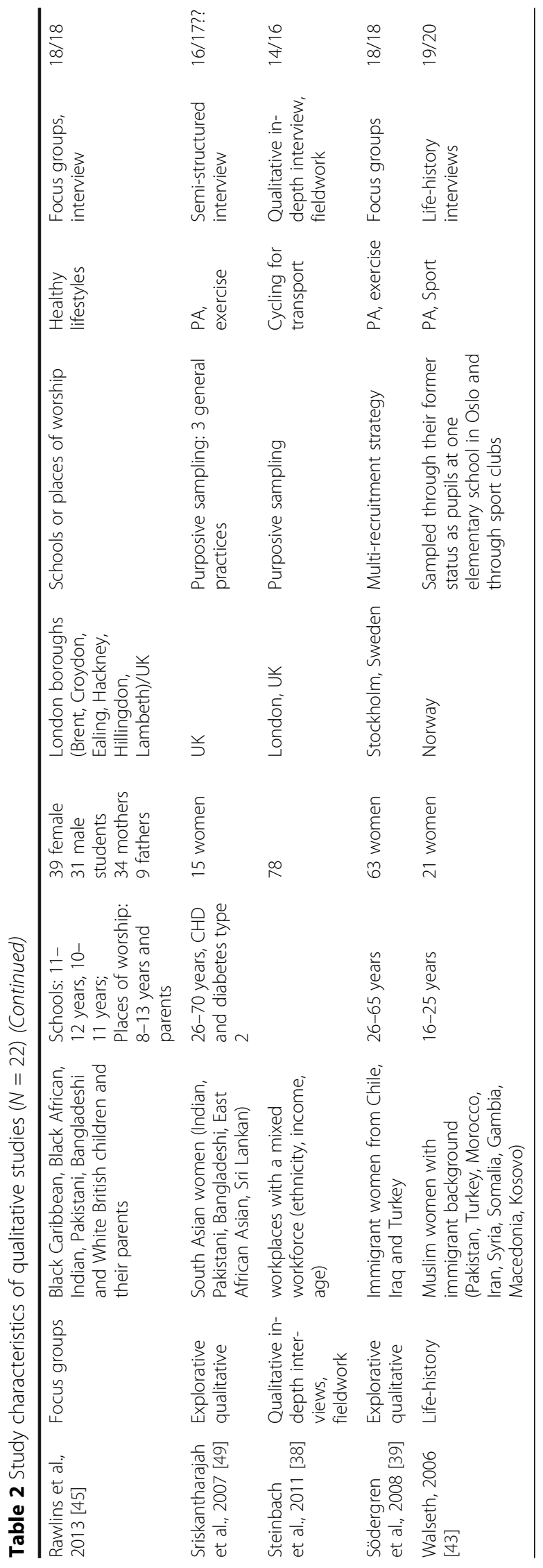


groups living in Europe (manuscript in preparation: [10]). For PA/SB the factors were grouped into eight clusters; Health \& health communication, Political environment, Social \& cultural environment, Psychosocial, Institutional environment, Physical environment \& accessibility, Social \& material resources and Migration context.

\section{Results}

\section{Description of included studies}

We identified 63 articles on PA and SB (41 based on quantitative studies; 22 on qualitative) among ethnic minority groups in Europe (Fig. 1). It should be noted that we in some cases have chosen to include more than one article from the same study, or research populations, when the articles address different issues, or mention different factors of PA or SB. The large majority of the studies were on PA $(n=59)$, while five focused on both PA and SB. No paper focused solely on SB. About half of the studies $(n=33)$ were conducted in the Western parts of Europe, most in the UK. A large number of the studies were conducted in the Nordic countries $(n=19)$. A few studies were comparative $(n=7)$, comparing two European countries or at least one European country with countries on other continents. Additional file 2 summarises description of the included studies.

We categorised the populations studied by country of origin, region, ethnicity or religion [see Additional file 2 and a more detailed division can be seen in Tables 1 and 2]. Most of the studies focused on more than one population. The main bulk of the studies focused on South Asian $(n=36)$, from India, Pakistan, or Bangladesh. These populations are especially important minority groups in the UK. Twelve studies focused on populations of Middle Eastern heritage, while nine studied African or Black African populations. Four studies focused on people of North African descent. The remaining populations studied were: South Americans $(n=3)$, East Africa, ns $(n=1)$, West Africans $(n=1)$. A few studies did not specify where the minority populations descended from $(n=8)$, but used such terms as 'other origin,' 'migrants', or 'Muslims'. 27 of the studies were either comparing ethnic minority groups and the majority populations, or studies of the general populations indicating ethnic background. Two studies were on indigenous population in the Nordic countries (Sami: $n=2)$ ).

Most of the 63 studies included both men and women $(n=45)$. Of the remaining studies 14 focused on women and 4 on men. Adults were the most studied group $(n=35)$. The second largest group was children $(n=13)$, followed by adolescents $(n=6)$, young older adults $(n=4)$, and older adults $(n=2)$. Three studies focused on the general population, and age was not specified. The number of participants ranged from 92 to 14,485 (of which 1498 were immigrants) in the quantitative studies and 15-202 (a few focus group studies had high numbers of participants) in the qualitative studies.

\section{Factors influencing physical activity/sedentary behaviour} We identified 183 distinct factors (Table 3) influencing PA and SB among ethnic minority groups in Europe. Of the identified factors, 60 were identified in qualitative studies and 54 were identified through quantitative studies only. The remaining factors were identified in both qualitative and quantitative studies, though many were predominantly identified through qualitative studies. The factors were often described as facilitators or barriers to PA and SB. Most factors were assigned to the Social \& cultural environment cluster ( $n=55$ factors), followed by Psychosocial $(n=39)$, Physical environment \& accessibility $(n=33)$, Migration context $(n=15)$, Institutional environment $(n=14)$, Social \& material resources $(n=12)$, Health \& health communication $(n=12)$, and finally Political environment $(n=3)$. The factors in the smallest cluster "Political environment" were identified only in qualitative studies. Qualitative studies were also strongly represented in most of the other clusters, but quantitative studies dominated the clusters "Migration context" and "Social \& material resources".

There were some similarities and differences in clusters of factors influencing PA and SB across different ethnic minority groups (Table 3 ). For example, "Social and cultural environment", "Social and material resources" and "Migration context" were identified in many of the study populations and studies with mixed populations. Many of the factors in the clusters "Physical environment and accessibility", "Psychosocial and institutional environment" and "Health and health communication" were common in studies conducted among South Asians, the largest group in the review, but also African, Caribbean, Turkish and mixed. Qualitative studies that focused on Muslim groups were represented in most clusters except "Migration context" and "Social and material resources".

\section{Clusters}

\section{"Social \& cultural environment"}

The "Social \& cultural environment" cluster included most factors ( 55 factors). The most cited factor was gender [5, 18-39] (Table 2). Gender, as a factor that influences PA and SB in ethnic minority groups, span different notions, and included cultural and religious notions of gender, moralities related to gender, and as well as social expectations and gender roles.

Factors related to religion recurred often as well. These included: religious requirements $[20,26,31,34$, $37,40-43]$ and religion and culture $[5,18,20,26,43-$ 46]. Following the factors were, commitments within family $[20,24,27,31,39,40,47,48]$ and modesty [5, 23, $26,27,31,38,40,49]$. Other factors related to gender, 


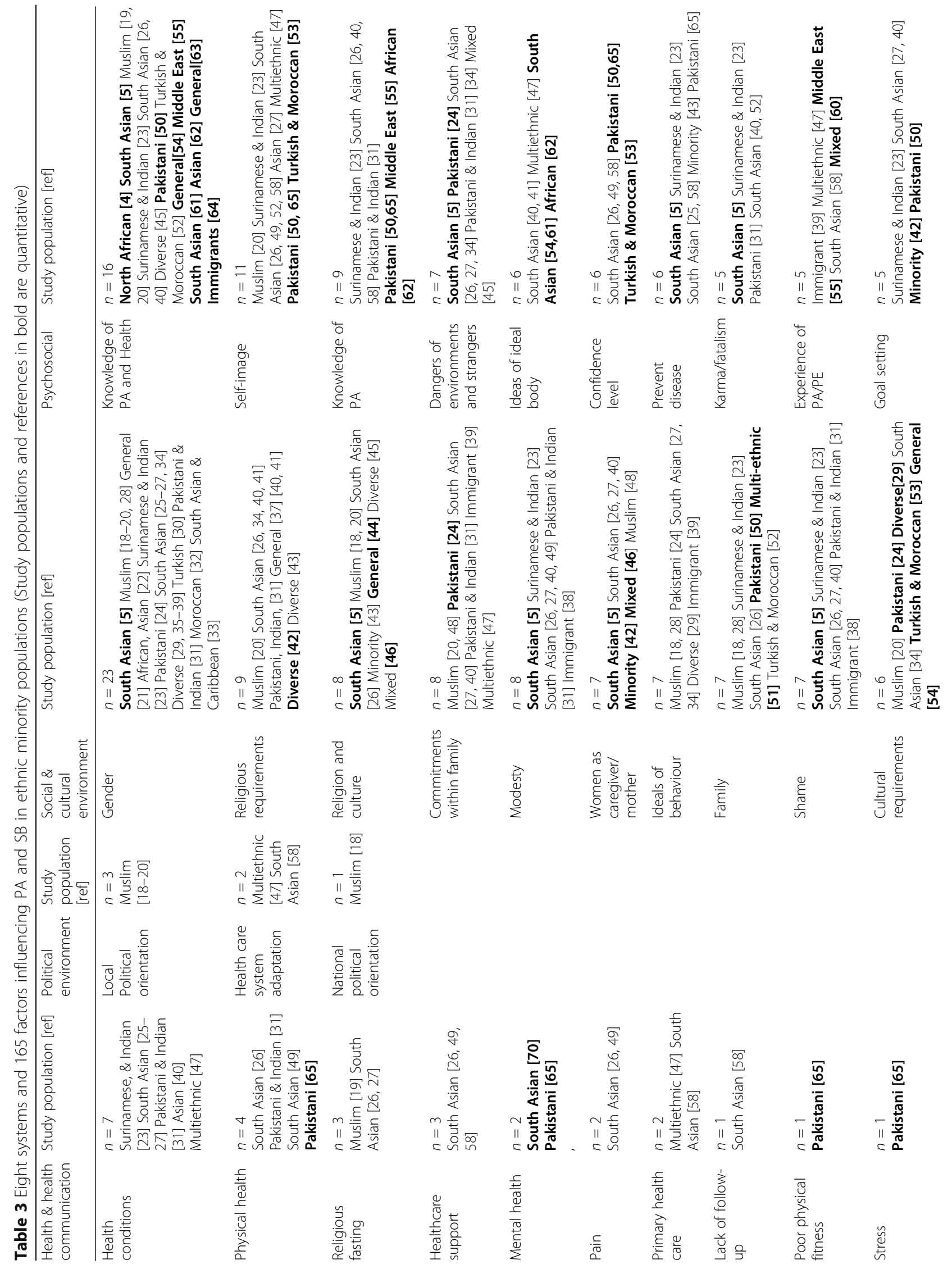




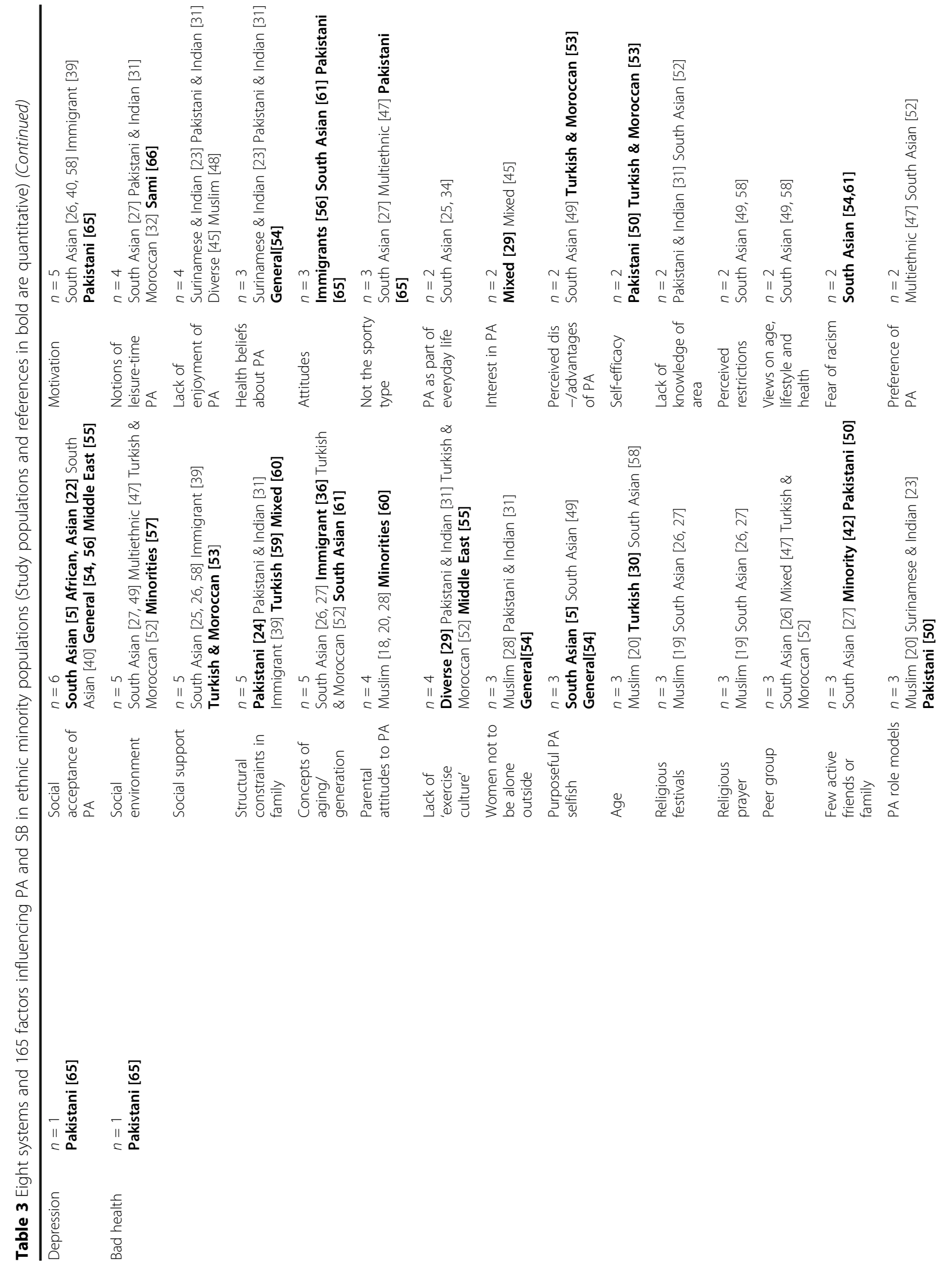




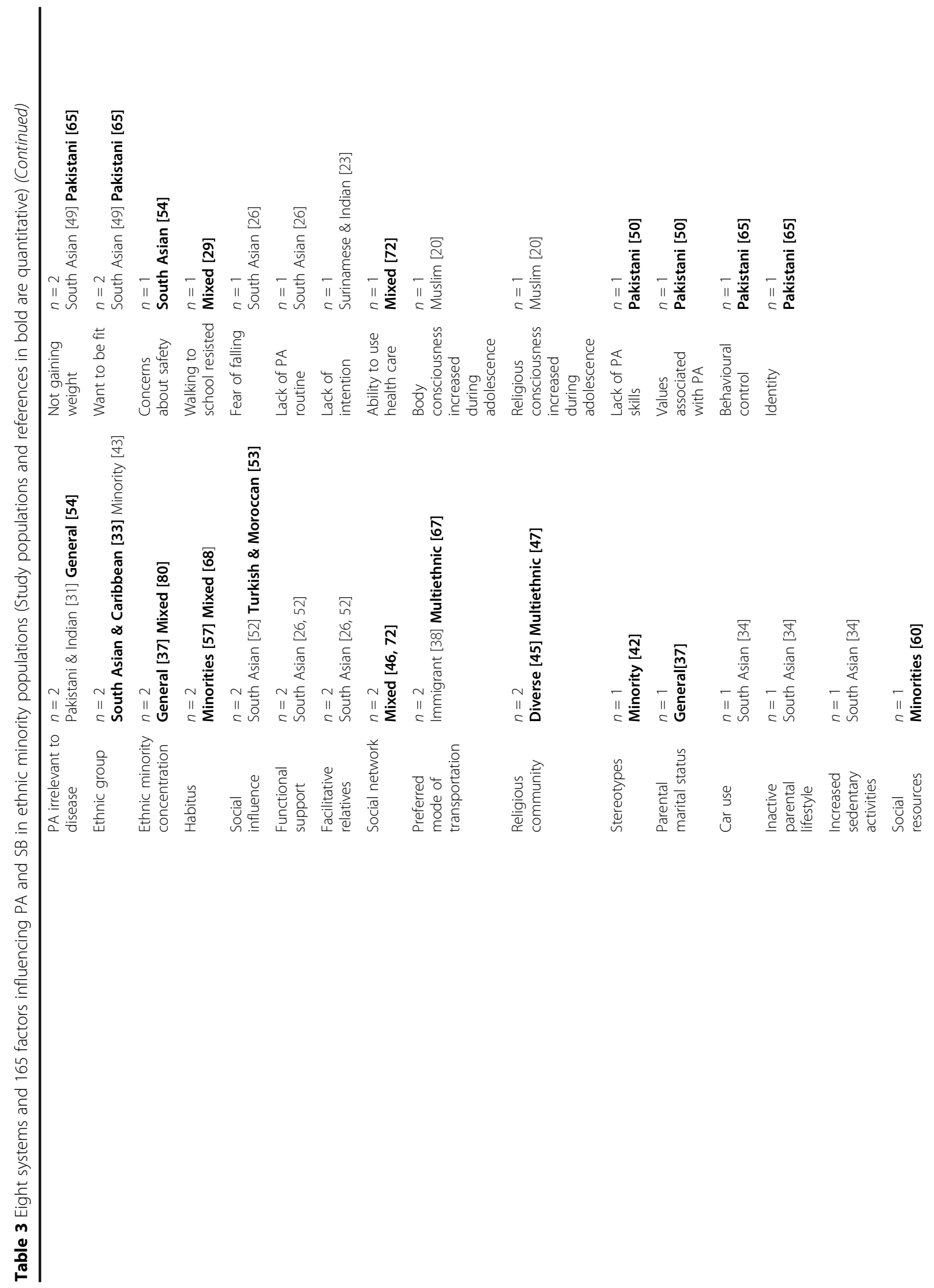




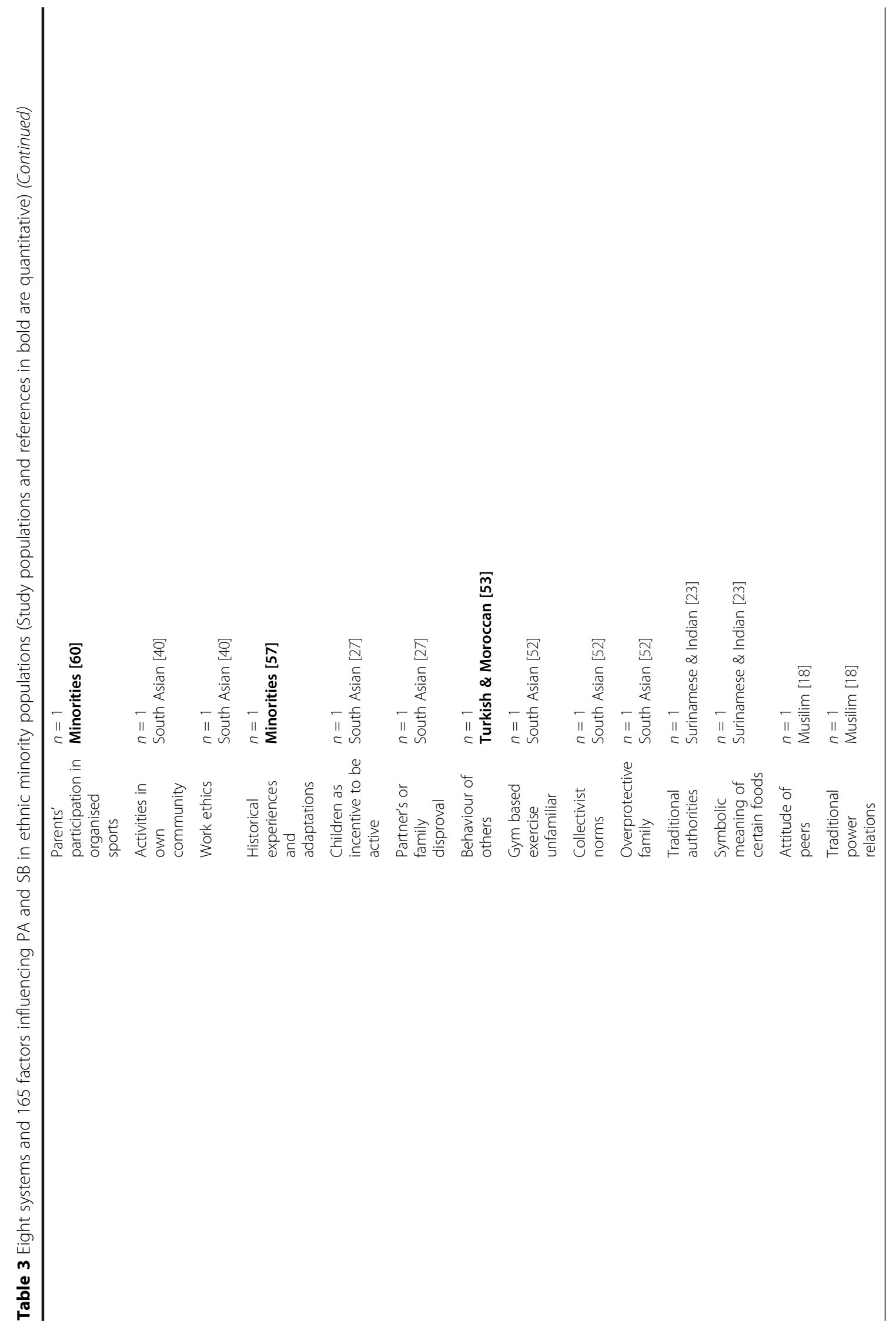




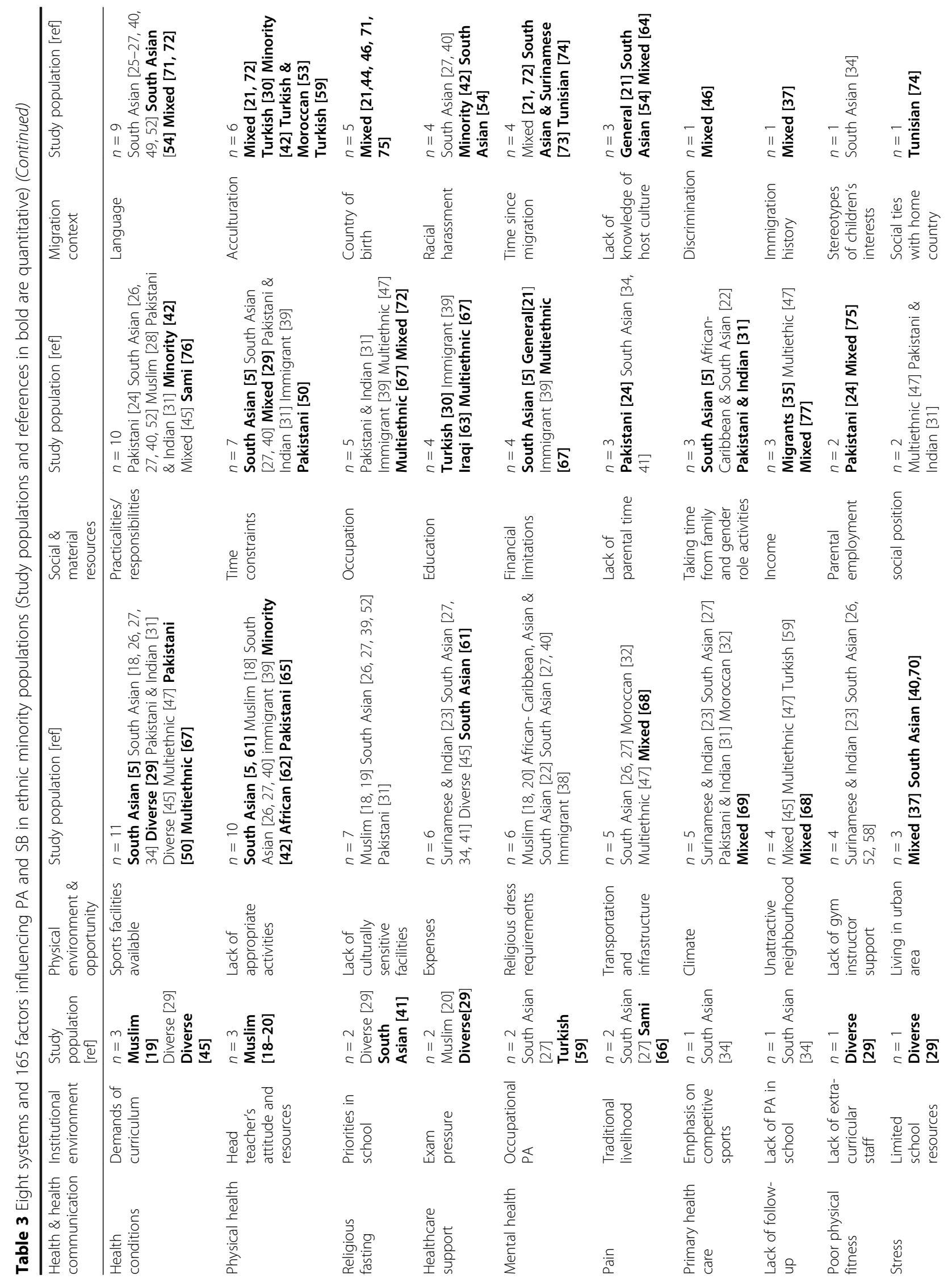




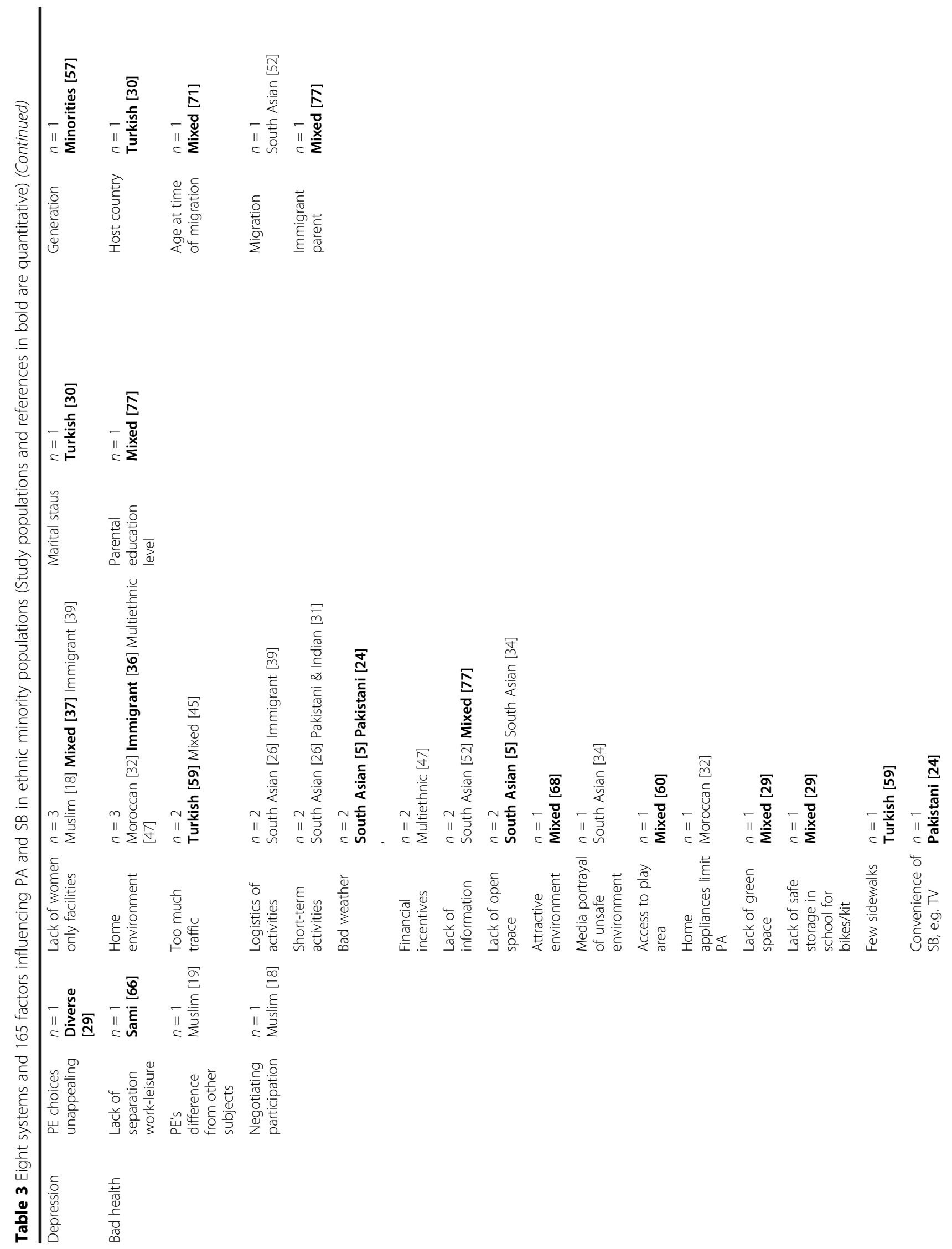




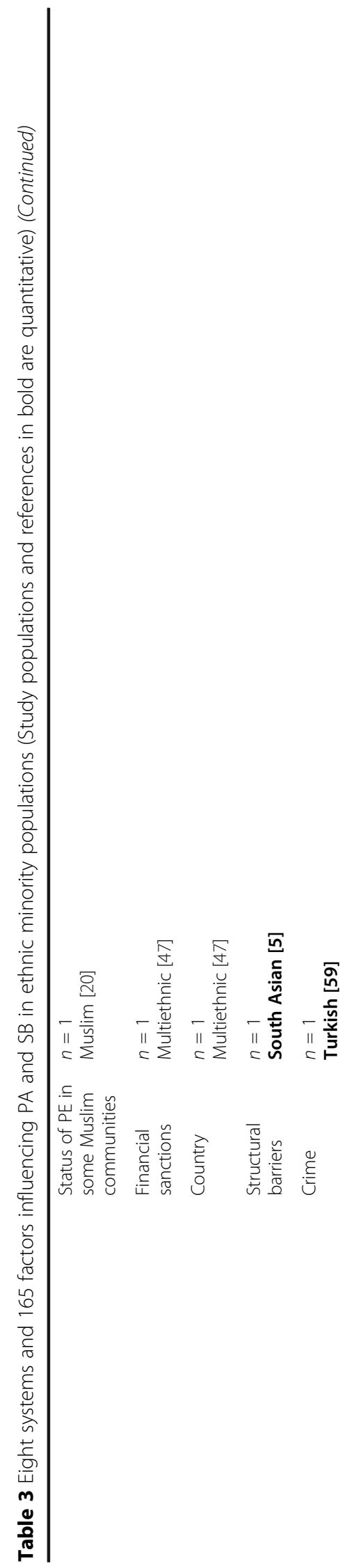


family, religious and cultural issues were: women as caregiver/mother [5, 26, 27, 40, 42, 46, 48], ideals of behaviour $[18,24,27-29,34,39]$, family $[18,23,26,28$, 50-52] and shame [5, 23, 26, 27, 31, 38, 40]. The broader factors, religious requirements and religion and culture, encompassed religious requirements of dressing, as well as degree of religiosity or piety.

The cluster also included factors relating to cultural requirements [20, 24, 29, 34, 53, 54], social acceptance of PA [5, 22, 40, 54-56], social environment [27, 47, 49, $52,57]$, social support $[25,26,39,53,58]$, structural constraints in family $[24,31,39,59,60]$ and concepts of age/aging [26, 27, 36, 52, 61]. Cultural requirements included ideals of behaviour and morality, as well as commitments of attending religious ceremonies and notions of the relationship between genders. The factor social acceptance of PA covered more specific factors. For instance, Babakus et al. [5] described that education about Muslim faith can be a motivating factor as PA is seen as central to the Muslim way of life.

Some studies included the factors: parental attitudes to PA $[18,20,28,60]$, lack of 'exercise culture' [29, 31, 52, $55]$, women not to be alone outside [28, 31, 54], purposeful PA selfish [5, 49,54], age [20, 30, 58], religious festivals $[19,26,27]$, religious prayer $[19,26,27]$, peer group $[26,47,52]$, few active friends or family [27, 42, $50]$, and PA role models $[20,23,50]$. The remaining factors were found in only one or two studies. Many studies were on South Asian and Muslim women, but also other groups.

\section{"Psychosocial"}

The "Psychosocial" cluster included individually held factors. Knowledge of PA and health $[4,5,19,20,23,26$, $40,45,50,52,54,55,61-64]$, the factor included in most studies, covers knowledge, notions and ideas about what constitutes PA and the relationship between health and PA. Self-image [20, 23, 26, 27, 47, 49, 50, 52, 53, 58, 65], which encompasses different notions of who one is and should be, was mainly reported in qualitative studies.

The cluster also included factors such as knowledge of PA [23, 26, 31, 40, 50, 55, 58, 62, 65], dangers of environment and strangers [5,24,26,27,31,34, 45], ideas of ideal body [40, 41, 47, 54, 61, 62], confidence level [26, $49,50,53,58,65]$, prevent disease [5, 23, 25, 43, 58, 65], notions of karma/fatalism [5, 23, 31, 40, 52], experience of PA/PE $[39,47,55,58,60]$, goal setting $[23,27,40,42$, $50]$, motivation $[26,39,40,58,65]$, notions of leisuretime PA [27, 31, 32, 66], lack of enjoyment of PA [23, $31,45,48]$, health beliefs about PA [23, 31, 54], attitudes $[56,61,65]$, not the sporty type [27, 47, 65]. The remaining factors were found in only one or two articles. Study populations were mixed in relation to gender.
"Physical environment \& accessibility"

Sports facilities available $[5,18,26,27,29,31,34,45,47$, $50,67]$, lack of appropriate activities $[5,18,26,27,39$, $40,42,61,62,65]$ and lack of culturally sensitive facilities $[18,19,26,27,31,39,52]$ were the most widely cited PA and SB factors in this cluster. Lack of appropriate activities and lack of culturally sensitive facilities were often interwoven with gender issues, as for instance whether or not an activity was appropriate for women or whether women and men could exercise separately. Lack of women only facilities [18, 37, 39] was mentioned as a factor as well.

This cluster also included expenses [23, 27, 34, 41, 45, 61], religious dress requirements [18, 20, 22, 27, 38, 40], transportation and infrastructure [26, 27, 32, 47, 68], climate [23, 27, 31, 32, 69], unattractive neighbourhood $[45,47,59,68]$, lack of gym instructor support [23, 26, $52,58]$, living in urban area $[37,40,70]$, and home environment [32, 36, 47]. The remaining 20 factors were found in only one or two studies. Study populations were mixed in relation to gender.

\section{"Migration context"}

The main factor in the "Migration context" cluster was language [25-27, 40, 49, 52, 54, 71, 72], meaning a lack or poor knowledge of the language of the host country. Language was seen as having an impact on PA and SB in several ways. As a barrier to get access of knowledge about PA, SB and health, as well as information on when and where to do PA. The second most cited factor in this cluster was acculturation $[21,30,42,53,59,72]$, meaning the ways minorities become part of the wider society. "Acculturation" was often related to other factors such as time since migration [21, 72-74], generation [57], and immigration history [37].

Other factors grouped under this cluster included country of birth [21, 44, 46, 71, 75] (implying cultural background, but also host country's attitudes towards immigrants from a specific country), racial harassment $[27,40,42,54]$, and lack of knowledge of host culture $[21,54,64]$. The remaining seven factors were only found in only study each. Study populations were mixed in relation to gender.

\section{"Institutional environment"}

The cluster "Institutional environment" included factors related to school and work environment. In schools, demands of curriculum $[19,29,45]$ were seen as having an impact on PA both because schools prioritise other subjects than physical education (PE) [19], and because parents value homework over leisure time PA [45]. Head teacher's attitude and resources [18-20] also had an impact on PA. Teachers could choose whether or not to let ethnic minority pupils participate on their own terms, 
i.e. separating boys and girls, or allowing ethnic dress requirements, and because limited resources can make it harder to prioritise PA or extra-curricular activities. Other factors included priorities in school $[29,41]$, exam pressure [20, 29], occupational PA [27, 59] and traditional livelihood [27, 66]. Many studies focused on Muslim women and girls.

\section{"Social and material resources"}

Practicalities/responsibilities [24, 26-28, 31, 40, 42, 45, $52,76]$ were the most cited factors assigned to the cluster "Social and material resources." The cluster also consisted of time constraints [5, 27, 29, 31, 39, 40, 50], occupation $[31,39,47,67,72]$, education $[30,39,63$, 67], financial limitations [5, 21, 39, 67], lack of parental time [24, 34, 41], taking time from family and gender role activities [5, 22, 31], and income [35, 47, 77]. The remaining four factors were only reported in one or two studies. Study populations were mixed in relation to gender.

\section{"Health and health communication"}

This cluster included health conditions [23, 25-27, 31, $40,47]$, physical health [26, 31, 49, 65], religious fasting $[19,26,27]$, healthcare support [26, 49, 58], mental health $[65,70]$, pain $[26,49]$ and primary health care $[47,58]$. The remaining five factors were found in one article. The majority of studies focused on women.

\section{"Political environment"}

This is the cluster with the smallest number of factors: local political orientation [18-20], health care system adaptation $[47,58]$ and national political orientation [18]. Local and national political orientation were seen as having an impact on PA and SB in ethnic minority groups by the willingness to make adaptations to better suit minority populations. Health care system adaptation had similar impact on PA and SB, by conveying the message of PA and health in ways adapted to ethnic minority groups. Many studies focused on Muslim women and girls.

\section{Discussion}

The main aims of this study were to identify factors influencing physical activity and sedentary behaviour among ethnic minority groups living in Europe, uncover gaps in the literature, and to suggest priorities for future research. The review extracted 183 factors that were grouped into eight clusters. The "Social \& cultural environment" and "Psychosocial" were the clusters containing most factors.

An important finding of our study was that cultural and religious issues, in particular those related to gender issues, were recurring factors across the clusters. Among these were cultural ideas of the body. Ideas of healthy ways to move and appropriateness of exposing parts of the body to perform physical activity are culturally loaded and tend to vary according to age, gender and roles/responsibilities within the family. For instance, research among adolescents has shown that consciousness of one's own body and religious consciousness evolve during these years, and affect individual's involvement and meaning of PA [20]. Importantly, cultural and religious factors can both hinder and facilitate PA. For example, religious requirements on how to dress $[18,20$, $22,27,38,40$ ] and limitations on spending time with the opposite gender might hinder PA, but staying fit and walking to religious sites for prayer were sometimes religious merits [27]. Other cultural issues involved ideas of femininity [43], religious or traditional ideas of ideal behaviour and attitudes related to leisure time. Moreover, there was the impact of lack of gender-segregated facilities [27, 39, 61]. Being female is not necessarily a barrier to PA in Muslim populations, but if there are no ways in which women can be active without encountering men, this might lead to women being less active. Not being able to separate between Islamic ideals (which is not against women being active) and particular cultural traditions of some Muslim populations (which might be counter to PA among women) was one factor of PA identified [20]. Therefore, understanding how religion and culture or tradition interact with other factors requires more in-depth study.

In stating that there are important factors to be found in the interchange between "gender," "culture" and "religion," it is vital to keep in mind that many of the studies have been undertaken among Muslim groups, thus the findings of this review reflect the populations studied.

The study showed that there were also many factors related to knowledge and information across the clusters, such as lack of knowledge and information about PA and the relationship between health and PA [4, 5, 20, $23,26,31,40,45,50,55,58,61-65]$. Lack of knowledge and information about facilities in own community [31, 52, 77] were reported as well. Other emerging factors were lack of knowledge of new culture and familiarity with wider community [21, 54, 64].

Language capabilities [25-27, 40, 49, 52, 54, 71, 72] had impact on access to information and knowledge about opportunities for PA in local areas and recommendations for PA. Additionally, lack of fluency in language can make it harder to follow PA classes/courses.

The review indicated that ethnic minority groups are influenced by many of the same factors as majority populations, such as age, knowledge and physical environment $[15,78,79]$, but the processes underlying these factors (culture, religion, lay models etc.) were distinctive for ethnic minority groups. Specific factors for ethnic minority groups, such as cultural, religious, and/or traditional values, perceptions and ideas (associated with PA, 
sedentary behaviour and body) emerged. The review showed that there are divergent notions of what constitutes physical activity and exercise [27, 31, 32, 66] and how different activities might relate to health $[4,5,20,26$, $31,40,45,50,52,54,55,61,64]$. Culturally dependent knowledge, notions and ideas of the physical activity and health and how they are related were among the most important reasons for not being active. For instance, some ethnic groups viewed physical activity as detrimental for health, especially for women and the elderly. Thus, it is important to gain knowledge about the cultural ideas of physical activity in different ethnic minority groups, but also to develop culturally sensitive information about the health benefits of physical activity, to create effective interventions and policies.

\section{Strengths and limitations of the review}

This is the first systematic mapping review that has described factors influencing PA and SB among a diverse ethnic minority groups living in Europe. One difference between this review and earlier reviews is the method used in synthesizing the findings. In this review, we have used a novel categorization based on clustering factors. This approach transcends existing models by aiming at better capturing the complexity of the system of factors influencing behaviour $[10,13]$. Another strength of the study is that the study design included both quantitative and qualitative published European literature. The review indicated that quantitative and qualitative studies contribute to our understanding of PA and SB among ethnic minority groups by providing somewhat different sets of factors. Qualitative studies used more explorative designs about PA and SB in ethnic groups, and yielded richer and more detailed information about the interrelationship between factors and clusters. One challenge in this review is that the categorisation of ethnic groups varied widely between the different studies, making it difficult to compare the findings.

The review indicates that there is more evidence for the role of individual level factors like gender, knowledge of PA and health, and health conditions compared with environmental factors. This tendency might in part stem from the place of the individual in the "Western" society. However, it is also important to note that the number of studies citing each factor is not necessarily an indication of the importance of those factors. Rather, it tells us is how many studies have selected to focus on each factor. For instance, the fact that few factors related to political environment have been found does not mean that environmental factors are not important, but there has been less focus on them.

The importance of religion and gender issues may be due to the fact that many studies have been conducted on populations with Islamic faith. In order to further explore factors related to PA and SB among ethnic minorities, more studies on populations of different religious affiliation (for example, Hinduism, Catholicism) are needed.

\section{Implication of the findings}

The literature review indicates that both PA and SB among ethnic minority groups in Europe are influenced by a wide variety of factors that are related and cut across different clusters of influence. Our findings support the need of adopting a systems-based framework [10] to capture the complexity of PA and SB among ethnic minority populations. Studies adopting a qualitative research design provided a richer understanding of underlying factors related to PA and SB.

The literature review indicates that comparative studies are limited. One recommendation from the review is that there is a need for systematic comparative research across Europe to shed light on the processes in which similar factors drive PA and SB behaviours on specific groups in different national and regional settings. Most studies included population from South Asia and Muslim populations, but as the European population is changing there is the need to further research on other ethnic groups, for example, asylum seeking groups from Somalia, Eritrea and Syria.

The majority of the studies were conducted in the Western part of Europe, mainly in the UK, and the Nordic countries. This was reflected in the groups included in the review, which do not represent the diversity of ethnic minority groups and religious populations living in Europe. This calls for a broadening of the research scope to include all parts of Europe.

Finally, the literature review indicates that there are very few studies on SB among ethnic minority groups in Europe. SB is a fairly new area of research and thus methods may not have not been adapted to ethnic minorities. As the relevance of SB for health outcomes is increasingly documented [15, 78], further research on this topic needs to specifically address ethnic minority groups.

\section{Conclusions}

This systematic review identified 183 factors influencing PA and SB across some ethnic minority and religious groups (Muslim) living in Europe. Factors were grouped into eight clusters following a data driven approach. The most recurrent factors (gender, religion, cultural requirements and knowledge) were part of the clusters 'Social \& cultural environment' and 'Psychosocial'.

The review indicated that there are several gaps in the literature related to the ethnic minority populations studied, the countries where the studies have been conducted, paucity of comparative studies and lack of attention towards SB. 
The review showed that there are some specific factors influencing PA and SB among ethnic minority groups. It is important to further address these factors in order to plan and implement effective interventions.

\section{Additional files}

Additional file 1: Systematic search strategy. (DOCX 83 kb)

Additional file 2: Characteristics of quantitative and qualitative studies. (DOCX $17 \mathrm{~kb})$

\section{Abbreviations}

DEDIPAC KH: Determinants of Diet and Physical Activity Knowledge Hub; NCDs: Non-communicable diseases; PA: Physical Activity; SB: Sedentary Behaviour

\section{Acknowledgements}

The authors wish to thank the entire Work Package 2.4 team of the DEDIPAC KH.

\section{Funding}

The preparation of this paper was supported by the Determinants of Diet and Physical Activity (DEDIPAC) Knowledge Hub. The DEDIPAC project is supported by the Joint Programming Initiative 'Healthy Diet for a Healthy Life', a research and innovation initiative of European Union member states and associated countries. The funding agencies supporting this work are (in alphabetical order of participating Member State): Norway: Norwegian Research Council; The Netherlands: the Netherlands Organisation for Health Research and Development; The United Kingdom: The Medical Research Council.

\section{Availability of data and materials}

Not applicable.

\section{Authors' contributions}

All authors conceptualised and designed the study. LJL, LT, GrR and GR screened and extracted the data. LJL drafted the manuscript. All authors reviewed draft versions of the manuscript and provided suggestions and critical feedback. All authors have made a significant contribution to this manuscript and approved the final manuscript.

Ethics approval and consent to participate Not applicable.

\section{Consent for publication}

Not applicable.

\section{Competing interests}

The authors declare that they have no competing interests.

\section{Author details}

${ }^{1}$ Norwegian School of Sport Sciences, Oslo, Norway. ${ }^{2}$ Department of Nursing and Health Promotion, Faculty of Health Sciences, Oslo and Akershus University College of Applied Sciences, Oslo, Norway. ${ }^{3}$ Department of Public Health, Academic Medical Centre, University of Amsterdam, Amsterdam, the Netherlands. ${ }^{4}$ Public Health Section, School of Health and Related Research, University of Sheffield, Sheffield, UK. ${ }^{5}$ Department of Nutrition, University of Oslo, Oslo, Norway. ${ }^{6}$ Consumption Research Norway - SIFO, Oslo and Akershus University College of Applied Sciences, Oslo, Norway.

Received: 5 December 2016 Accepted: 12 July 2017

Published online: 24 July 2017

\section{References}

1. Crepso CJ, Smit E, Andersen RE, Carter-Pokras O, Ainsworth BE. Race/ethnicity, social class and their relation to physical inactivity during leisure time: results from the third National Health and nutrition examination survey, 1988-1994. Am J Prev Med. 2000;18(1):46-53.
2. Glover-Larsen P, Mcmurray RG, Popkin BM. Adolescent physical activity and inactivity vary by ethnicity: the national longitudinal study of adolescent health. J Pediatr. 1999;135(3):301-6.

3. Whitt-Glover MC, Taylor WC, Floyd MF, Yore MM, Yancey AK, Matthews CE. Disparities in physical activity and sedentary behaviors among US children and adolescents: prevalence, correlates, and intervention implications. J Public Health Policy. 2009;30(Suppl 1):S309-34

4. Gualdi-Russo E, Zaccagni L, Manzon V, Masotti S, Rinaldo N, Khyatti M. Obesity and physical activity in children of immigrants. Eur J Pub Health. 2014;24(Suppl. 1):40-6.

5. Babakus W, Thompson J. Physical activity among south Asian women: a systematic, mixed-methods review. Int J Behav Nutr Phys Act. 2012;9(1):150.

6. Eurostat. Migration and migrant populations. 2017. http://ec.europa.eu/ eurostat/statistics-explained/index.php/Migration_and_migrant_population_ statistics. Accessed 24 Apr 2017.

7. Eurostat. Asylum statistics. 2017. http://ec.europa.eu/eurostat/statisticsexplained/index.php/Asylum_statistics. Accessed 24 Apr 2017.

8. WHO. Migration and health: Key issues. 2017. http://www.euro.who.int/en/ health-topics/health-determinants/migration-and-health/migrant-health-in-theeuropean-region/migration-and-health-key-issues. Accessed 24 Apr 2017.

9. Lakerveld J, Van Der Ploeg HP, Kroetze W, Ahrens W, Allais O, Andersen LF, et.al. Towards the integration and development of a cross-European research network and infrastructure: the DEterminants of Dlet and physical ACtivity (DEDIPAC) knowledge hub. Int J Behav Nutr Phys Act 2014;11:143.

10. Holdsworth M, Nicolaou M, Langøien LJ, Osei-Kwasi HA, Chastin SFM, Stok FM, et al. Developing a system-based framework of the factors influencing dietary and physical activity behaviours in ethnic minority populations living in Europe - A DEDIPAC study (Manuscript under review). 2016.

11. Grant MJ, Booth A. A typology of reviews: and analysis of 14 review types and associated methodologies. Health Inf Libr J. 2009;26:91-108.

12. Kmet LM, Lee RC, Cook LS. Standard quality assessment criteria for evaluating primary research papers from a variety of fields. Edmonton: Alberta Heritage Foundation for Medical Research (AHFMR). AFHMR - HTA Initiative \#3. 2004.

13. Osei-Kwasi HA, Nicolaou M, Powell K, Terragni L, Maes L, Stronks K, et al. Systematic mapping review of the factors influencing dietary behaviour in ethnic minority groups living in Europe: a DEDIPAC study. Int J Behav Nutr Phys Act. 2016;18:85.

14. Trochim WM. An introduction to concept mapping for planning and evaluation. Eval Program Plann. 1989;12(1):1-16.

15. Chastin SF, Buck C, Freiberger E, Murphy M, Brug J, Cardon G, et al. Systematic literature review of determinants of sedentary behaviour in older adults: a DEDIPAC study. Int J Behav Nutr Phys Act. 2015;12(1):127.

16. Condello G, Ling FCM, Bianco A, Chastin S, Cardon G, Ciarapica D, et al. Using concept mapping in the development of the EU-PAD framework (European physical activity determinants across the life course): a DEDIPACstudy. BMC Public Health. 2016;16:1145.

17. Stok FM, Renner B, Hoffmann S, Volkert D, Boeing H, Ensenauer R, et al. The DONE framework: Creating, evaluating and updating of an interdisciplinary, dynamic framework 2.0 of determinants of nutrition and eating (Manuscript under review). 2016.

18. Benn T, Pfister G. Meeting needs of Muslim girls in school sport: case studies exploring cultural and religious diversity. Eur J Sport Sci. 2013;13(5):567-74.

19. Dagkas S, Benn T. Young Muslim women's experiences of Islam and physical education in Greece and Britain: a comparative study. Sport Educ Soc. 2006;11(1):21-38.

20. Dagkas S, Benn T, Jawad H. Multiple voices: improving participation of Muslim girls in physical education and school sport. Sport Educ Soc. 2011;16(2):223-39.

21. Dawson AJ, Sundquist J, Johansson S-E. The influence of ethnicity and length of time since immigration on physical activity. Ethn Health. 2005;10(4):293-309.

22. Garduno-Diaz S, Khokhar S. Assessment and comparison of diet quality and physical activity of African-Caribbean, south Asian and Caucasian groups in the UK. Int J Food Sc Nutr Diet. 2013;2(5):45-50.

23. Hendriks A-M, Gubbels JS, Jansen MWJ, Kremers SPJ. Health beliefs regarding dietary behavior and physical activity of Surinamese immigrants of Indian descent in The Netherlands: a qualitative study. ISRN Obes. 2012;2012:903868.

24. Hornby-Turner YC, Hampshire KR, Pollard TM. A comparison of physical activity and sedentary behaviour in 9-11 year old British Pakistani and white British girls: a mixed methods study. Int J Behav Nutr Phys Act. 2014;11:74. 
25. Horne M, Skelton DA, Speed S, Todd C. Attitudes and beliefs to the uptake and maintenance of physical activity among community-dwelling south Asians aged 60-70 years: a qualitative study. Public Health. 2012;126(5):417-23.

26. Horne M, Skelton DA, Speed S, Todd C. Perceived barriers to initiating and maintaining physical activity among south Asian and white British adults in their 60s living in the United Kingdom: a qualitative study. Ethn Health. 2013;18(6):626-45.

27. Johnson MRD. Perceptions of barriers to healthy physical activity among Asian communities. Sport Educ Soc. 2000;5(1):51-70.

28. Kay T. Daughters of Islam: family influences on Muslim young women's participation in sport. Int Rev Sociol Sport. 2006;41(3-4):357-73.

29. Khunti K, Stone MA, Bankart J, Sinfield P, Pancholi A, Walke S, et al. Primary prevention of type-2 diabetes and heart disease: action research in secondary schools serving an ethnically diverse UK population. J Public Health. 2008;30(1):30-7.

30. Koca C, Lapa TY. Analysis of physical activity and acculturation among Turkish migrants in Germany and England. Percept Mot Skills. 2014;119(3):698.

31. Lawton J, Ahmad N, Hanna L, Douglas M, Hallowell N. 'I can't do any serious exercise': barriers to physical activity amongst people of Pakistani and Indian origin with type 2 diabetes. Health Educ Res. 2006;21(1):43-54.

32. Nicolaou M, Benjelloun S, Stronks K, van Dam RM, Seidell JC, Doak CM. Influences on body weight of female Moroccan migrants in the Netherlands: a qualitative study. Health Place. 2012;18(4):883-91.

33. Owen CG, Nightingale CM, Rudnicka AR, Cook DG, Ekelund U, Whincup PH. Ethnic and gender differences in physical activity levels among 9-10-yearold children of white European, south Asian and African-Caribbean origin: the child heart health study in England (CHASE study). Int J Epidemiol. 2009;38(4):1082-93.

34. Pallan M, Parry J, Adab P. Contextual influences on the development of obesity in children: a case study of UK south Asian communities. Prev Med. 2012;54(3-4):205-11

35. Pudaric S, Sundquist J, Johansson SE. Major risk factors for cardiovascular disease in elderly migrants in Sweden. Ethn Health. 2000;5(2):137-50.

36. Reijneveld SA, Westhoff MH, Hopman-Rock M. Promotion of health and physical activity improves the mental health of elderly immigrants: results of a group randomised controlled trial among Turkish immigrants in the Netherlands aged 45 and over. J Epidemiol Community Health. 2003;57(6): 405-11.

37. Sagatun A, Kolle E, Anderssen SA, Thoresen M, Sogaard AJ. Three-year follow-up of physical activity in Norwegian youth from two ethnic groups: associations with socio-demographic factors. BMC Public Health. 2008;8. doi:10.1186/1471-2458-8-419

38. Steinbach R, Green J, Datta J, Edwards P. Cycling and the city: a case study of how gendered, ethnic and class identities can shape healthy transport choices. Soc Sci Med. 2011;72(7):1123-30.

39. Södergren M, Hylander I, Törnkvist L, Sundquist J, Sundquist K. Arranging appropriate activities: immigrant women's ideas of enabling exercise. Womens Health Issues. 2008;18(5):413-22.

40. Lucas A, Murray E, Kinra S. Heath beliefs of UK South Asians related to lifestyle diseases: A review of qualitative literature. J Obes. 2013;13. doi:10. 1155/2013/827674.

41. Pallan M, Parry J, Cheng KK, Adab P. Development of a childhood obesity prevention programme with a focus on UK south Asian communities. Prev Med. 2013;57(6):948-54.

42. Walseth K, Fasting K. Sport as a means of integrating minority women. Culture Sport Society. 2004;7(1):109-29.

43. Walseth K. Young Muslim women and sport: the impact of identity work. Leis Stud. 2006;25(1):75-94

44. Lindström M, Sundquist J. Immigration and leisure-time physical inactivity: a population-based study. Ethn Health. 2001;6(2):77-85.

45. Rawlins E, Baker G, Maynard M, Harding S. Perceptions of healthy eating and physical activity in an ethnically diverse sample of young children and their parents: the DEAL prevention of obesity study. J Hum Nutr Diet. 2013;26(2):132-44.

46. Södergren M, Sundquist K, Johansson S-E, Sundquist J, Hagströmer M. Associations between health-enhancing physical activity and country of birth among women. J Phys Act Health. 2010;7(5):613-21.

47. Beune E, Haafkens JA, Agyemang C, Bindels PJE. Inhibitors and enablers of physical activity in multiethnic hypertensive patients: qualitative study. J Hum Hypertens. 2010;24(4):280-90.

48. Pollard TM, Guell C. Assessing physical activity in Muslim women of south Asian origin. J Phys Act Health. 2012;9(7):970-6.
49. Sriskantharajah J, Kai J. Promoting physical activity among south Asian women with coronary heart disease and diabetes: what might help? Fam Pract. 2007;24(1):71-6.

50. Andersen E, Hostmark AT, Holme I, Anderssen SA. Intervention effects on physical activity and insulin levels in men of Pakistani origin living in Oslo: a randomised controlled trial. J Immigr Minor Health. 2013;15(1):101-10.

51. Edwardson CL, Gorely T, Musson H, Duncombe R, Sandford R. Does activityrelated social support differ by characteristics of the adolescent? J Phys Act Health. 2014;11(3):574-80.

52. Horne M, Tierney S. What are the barriers and facilitators to exercise and physical activity uptake and adherence among south Asian older adults: a systematic review of qualitative studies. Prev Med. 2012;55(4):276-84.

53. Hosper K, Nierkens V, van Valkengoed I, Stronks K. Motivational factors mediating the association between acculturation and participation in sport among young Turkish and Moroccan women in the Netherlands. Prev Med. 2008;47(1):95-100.

54. Yates T, Davies MJ, Gray LJ, Webb D, Henson J, Gill JMR, et al. Levels of physical activity and relationship with markers of diabetes and cardiovascular disease risk in 5474 white European and south Asian adults screened for type 2 diabetes. Prev Med. 2010;51(3-4):290-4.

55. Arvidson D, Leijon M, Sundquist J, Sundquist K, Lindblad U, Benet L. Crosscultural validation of a simple self-report instrument of physical activity in immigrants from the Middle East and native Swedes. Scand J Public Health. 2014;42(3):255-62

56. Drenowatz C, Steiner RP, Brandstetter S, Klenk J, Wabitsch M, Steinacker J, et al. Organized sports, overweight, and physical fitness in primary school children in Germany. J Obes. 2013;2013:935245.

57. Kumanyika S, Taylor WC, Grier SA, Lassiter V, Lancaster KJ, Morssink CB, Renzaho AMN. Community energy balance: a framework for contextualizing cultural influences on high risk of obesity in ethnic minority groups. Prev Med. 2012;55(5):371-81.

58. Horne M, Skelton D, Speed S, Todd C. The influence of primary health care professionals in encouraging exercise and physical activity uptake among white and south Asian older adults: experiences of young older adults. Patient Educ Couns. 2010;78(1):97-103.

59. Hosper K, Klazinga NS, Stronks K. Acculturation does not necessarily lead to increased physical activity during leisure time: a cross-sectional study among Turkish young people in the Netherlands. BMC Public Health. 2007; 7. doi:10.1186/1471-2458-7-230.

60. Nielsen G, Hermansen B, Bugge A, Dencker M, Andersen LB. Daily physical activity and sports participation among children from ethnic minorities in Denmark. Eur J Sport Sci. 2013;13(3):321-31.

61. Hayes L, White M, Unwin N, Bhopal R, Fischbacher C, Harland J, Alberti KGMM. Patterns of physical activity and relationship with risk markers for cardiovascular disease and diabetes in Indian, Pakistani, Bangladeshi and European adults in a UK population. J Public Health. 2002;24(3):170-8.

62. Gele AA, Mbalilaki AJ. Overweight and obesity among African immigrants in Oslo. BMC Res Notes. 2013;6(1):119.

63. Lecerof SS, Westerling R, Moghaddassi M, Östergren P-O. Health information for migrants: the role of educational level in prevention of overweight. Scand J Public Health. 2011;39(2):172-8.

64. Magnusson MB, Hulthen L, Kjellgren Kl. Obesity, dietary pattern and physical activity among children in a suburb with a high proportion of immigrants. J Hum Nutr Diet. 2005;18(3):187-94.

65. Andersen E, Hostmark AT, Lorentzen C, Anderssen SA. Low level of objectively measured physical activity and cardiorespiratory fitness, and high prevalence of metabolic syndrome among Pakistani male immigrants in Oslo, Norway. Norsk Epidemiologi. 2011;20(2):199-208.

66. Hermansen R, Njolstad I, Fonnebo V. Physical activity according to ethnic origin in Finnmark county, Norway. The Finnmark study. Int J Circumpolar Health. 2002;61(3):189-200.

67. de Munter JSL, Agyemang C, Brewster LM, Stronks K, van Valkengoed IGM. The association of leisure-time physical activity and active commuting with measures of socioeconomic position in a multiethnic population living in the Netherlands: results from the cross-sectional SUNSET study. BMC Public Health. 2012;12. doi:10.1186/1471-2458-12-815.

68. Saris C, Kremers S, Van Assema P, Hoefnagels C, Droomers M, et al. What moves them? Active transport among inhabitants of Dutch deprived districts. J Obes. 2013;2013:153973.

69. Kumar BN, Meyer HE, Wandel M, Dalen I, Holmboe-Ottesen G. Ethnic differences in obesity among immigrants from developing countries, in Oslo, Norway. Int J Obes. 2006;30:684-90. 
70. Williams ED, Stamatakis E, Chandola T, Hamer M. Assessment of physical activity levels in South Asians in the UK: findings from the Health Survey for England. J Epidemiol Community Health. 2010 doi:10.1136/jech.2009.102509.

71. Jönsson LS, Palmér K, Ohlsson H, Sundquist J, Sundquist K. Is acculturation associated with physical activity among female immigrants in Sweden? J Public Health. 2013;35(2):270-7.

72. Hansen AR, Ekholm O, Kjöller M. Health behaviour among non-western immigrants with Danish citizenship. Scand J Public Health. 2008;36(2):205-10.

73. De Munter JSL, Agyemang C, Van Valkengoed IGM, Bhopal R, Zaninotto P, Nazroo J, et al. Cross national study of leisure-time physical activity in Dutch and English populations with ethnic group comparisons. Eur J Pub Health. 2013;23(3):440-6.

74. Mejean C, Traissac P, Eymard-Duvernay S, Delpeuch F, Maire B. Influence of acculturation among Tunisian migrants in France and their past/present exposure to the home country on diet and physical activity. Public Health Nutr. 2009;12(6):832-41.

75. van Rossem L, Vogel I, Moll HA, Jaddoe WW, Hofman A, Mackenbach JP, Raat H. An observational study on socio-economic and ethnic differences in indicators of sedentary behavior and physical activity in preschool children. Prev Med. 2012;54(1):55-60.

76. Nilsson LM, Dahlgren L, Johansson I, Brustad M, Sjolander P, Van Guelpen B. Diet and lifestyle of the Sami of southern Lapland in the 1930s-1950s and today. Int J Circumpolar Health. 2011;70(3):301-18.

77. Besharat Pour M, Bergström A, Bottai M, Kull I, Wickman M, Håkansson N, et al. Effect of parental migration background on childhood nutrition, physical activity, and body mass index. J Obes. 2014;2014:1-10.

78. Brug J, Chinapaw M. Determinants of engaging in sedentary behavior across the lifespan; lessons learned from two systematic reviews conducted within DEDIPAC. Int J Behav Nutr Phys Act. 2015;12(1):1.

79. O'Donoghue G, Perchoux C, Mensah K, Lakerveld J, van der Ploeg H, Bernaards $C$, et al. A systematic review of correlates of sedentary behaviour in adults aged 18-65 years: a socio-ecological approach. BMC Public Health. 2016;16:163.

80. Molaodi OR, Leyland AH, Ellaway A, Kearns A, Harding S. Neighbourhood food and physical activity environments in England, UK: does ethnic density matter? Int J Behav Nutr Phys Act. 2012;9:75.

\section{Submit your next manuscript to BioMed Central and we will help you at every step:}

- We accept pre-submission inquiries

- Our selector tool helps you to find the most relevant journal

- We provide round the clock customer support

- Convenient online submission

- Thorough peer review

- Inclusion in PubMed and all major indexing services

- Maximum visibility for your research

Submit your manuscript at www.biomedcentral.com/submit 